\title{
Addresses of Reviewers
}

Dr. Vincent Barras, Institut Louis Jeantet d'histoire de la médecine, Case postale, 1211 Genève 4

Prof. Dr. Marcel H. Bickel, Medizinhistorisches Institut, Bühlstrasse 26, Postfach 139, 3000 Bern 9

Dr. Thomas Böni, Lenzstrasse 2, 8340 Hinwil

Prof. Dr. Urs Boschung, Medizinhistorisches Institut, Brühlstrasse 26, Postfach 139, 3000 Bern 9

Prof. Dr. Johann Jakob Burckhardt, Bergheimstrasse 4, 8032 Zürich

Dr. Urs Leo Gantenbein, Ackeretstrasse 16, 8400 Winterthur

Dr. Beat Glaus, ETH-Bibliothek, Wissenschaftshistorische Sammlung, Rämistrasse 101, 8092 Zürich

Prof. Dr. Felix Gutzwiller, Institut für Sozial- und Präventivmedizin, Universität Zürich, Sumatrastrasse 30, 3006 Zürich

Dr. Lies Huizink, General-Wille-Strasse 15, 8002 Zürich

PD Dr. Franz Josef Illhardt, Zentrum für Geriatrie und Gerontologie Freiburg, Lehener Strasse 88, D-79106 Freiburg

Prof. Dr. Robert Ineichen, 1, Ch. de l'Aurore, 1723 Marly

Dr. Hansruedi Isler, Neurologie, Universitätsspital, 8091 Zürich

Prof. Danielle Jacquart, 74, rue des Cévennes, F-75015 Paris

Dr. Hans Urs Keller-Schneider, Mühleackerstrasse 4, 4707 Deitingen

Prof. Dr. Huldrych M. Koelbing, Gotthardstrasse 65, 8002 Zürich

PD Dr. François Ledermann, Könizstrasse 3, 3000 Bern 21

Dr. Urs B. Leu, Sennpüntstrasse 5, 8303 Bassersdorf

Dr. Antonie M. Luyendijk-Elshout, Prins Bernhardlaan 60, NL-2341 KL Oegstgeest

Dr. Roger Mayer, 9, rue Michel-Chauvet, 1208 Genève

Prof. Philippe Mudry, Montolivet 28, 1006 Lausanne

Prof. Dr. Christian Müller, Herrengasse 23, 3011 Bern

PD Dr. Erwin Neuenschwander, Mathematisches Institut, Winterthurerstrasse 190, 8057 Zürich 
Dr. Thomas Nickol, Institut für Geschichte der Medizin, Krausenstrasse 14, D-06097 Halle

Dr. Iris Ritzmann, Dubsstrasse 30, 8003 Zürich

Prof. Dr. Gerhard Rudolph, Jamm-Strasse 4, D-77933 Lahr

PD Dr. Dr. Gottfried Schramm, Am Brunnenbächli 12, 8125 Zollikerberg

Dr. Roger Seiler, Medizinhistorisches Institut und Museum, Universität Zürich, Rämistrasse 71, 8006 Zürich

Dr. Verena Stadler-Labhart, Dokumentationsstelle für Universitätsgeschichte, Schönberggasse 15a, 8001 Zürich

Dr. Antoinette Stettler, Kirchenfeldstrasse 45, 3005 Bern

Prof. Dr. Jürg Wüst, Institut für med. Mikrobiologie, Universität Zürich, Gloriastrasse 30/32, Postfach, 8028 Zürich

Prof. Dr. Bernhard Zimmermann, Elisabeth-Mühlenweg-Strasse 13, D-78476 Allensbach 